\title{
DETERMINACIÓN DE LA EFICIENCIA Y EL COEFICIENTE TOTAL DE PÉRDIDAS DEL COLECTOR DE PLACA PLANA DE 2M², UTILIZANDO UN BANCO DE PRUEBAS SEGÚN LA NTP 399.400 EN AREQUIPA
}

\section{DETERMINATION OF THE EFFICIENCY AND THE TOTAL LOSS COEFFICIENT OF THE 2M ${ }^{2}$ FLAT PLATE COLLECTOR, USING A TEST BENCH ACCORDING TO NTP 399.400 IN AREQUIPA}

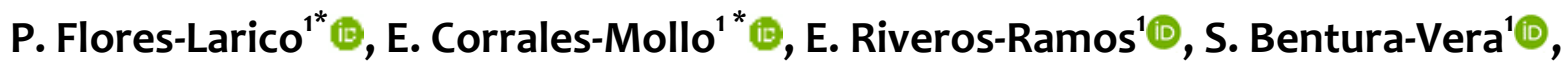 \\ C. Pedemonte-Grau' ${ }^{1}$, J. Milon-Guzman ${ }^{2}(0)$ \\ ${ }^{1}$ Universidad Nacional de San Agustín de Arequipa, Perú \\ ${ }^{2}$ Universidad Tecnológica del Perú-Arequipa, Perú
}

Recibido (Recieved): 21/02/2019 Aceptado (Accepted): 05/03/2020

\section{RESUMEN}

Arequipa es una ciudad con más de 70000 Sistemas de Calentamiento de Agua con Energía Solar (SCAES), existen más de 30 fabricantes de termas solares que no tienen laboratorios propios para poder determinar las características térmicas y la eficiencia. Se instaló un banco de pruebas para evaluar la eficiencia de los colectores solares de placa plana, bajo la norma técnica peruana NTP 399.400 2001. Tres colectores utilizados son de $2 \mathrm{~m}^{2}$ sirvieron para determinar el rango de valores de eficiencias máximas como del coeficiente global de pérdidas del sistema SCAES. Valores no determinados anteriormente con un módulo estandarizado.

Palabras-Clave: Energía Solar, Colector SCAES, Eficiencia

\section{ABSTRACT}

Arequipa is a peruvian south city with more than 70000 Solar Energy Water Heating Systems (know in spanish as SCAES). In this area there are more than 30 solar thermal manufacturers. They do not have laboratories to collet standarized data about characteristics and efficiency of their SCAES. In this research, a group of test was installed to evaluate the efficiency of three flat plat solar collectors $\left(2 \mathrm{~m}^{2}\right)$. This study was made under the Peruvian technical standard NTP 399.400 2001. As result, the range of maximum efficiency values and the global coefficient of losses of the SCAES was found.

Keywords: Solar Energy, SCAES Collector, Efficiency

* Corresponding author.:

E-mail: pfloresl@unsa.edu.pe 


\section{INTRODUCCIÓN}

En Alemania, USA (ASRHAE), Países de Europa (España-CENER), Brasil y otros existen laboratorios autorizados para evaluar los equipos solares los cuales han emitido normas para su construcción, evaluación y etiquetado energético.

Arequipa la ciudad de las "TERMAS SOLARES" tiene más de 70000 SCAES, las cuales fueron confeccionadas de manera técnica y artesanal, existiendo normativa en INDECOPI que debe aplicarse, para un mercado de 250000 hogares los cuales serán beneficiadas si se lograría certificar los SCAES (Sistemas de calentamiento de agua con energía solar).

Los fabricantes están produciendo aproximadamente 1000 termas solares por mes, lo que indica la aceptación de esta tecnología, que todavía no se replica en otro lugar del Perú. No existe en la ciudad entidades que fiscalicen la construcción, funcionamiento y venta de los SCAES.

En Arequipa, en la Universidad San Agustín se ha realizado trabajos de investigación en las áreas de física, mecánica y metalurgia y se ha logrado consolidar el Centro de Energía Renovables que agrupa a las áreas mencionadas.

Para ello debe existir una entidad que cuente con un laboratorio equipado con los diferentes equipos de medición que sería dirigido por el CER-EE-UNSA.

En el concurso de Proyectos de Investigación Básica y Proyectos de Investigación Aplicada 2016-02, UNSACONCYTEC-CIENCIA ACTIVA, se presentó el proyecto "Estudio experimental del desempeño de sistemas de calentamiento de agua con energía solar mediante un módulo estandarizado", nivel inicial, el cual fuimos ganadores con el financiamiento de un monto de S/100000,00 nuevos soles, a realizarse en 15 meses.

\section{ANTECEDENTES}

En el XXII Simposio Peruano de Energía Solar y del Ambiente (SPES) realizado en la ciudad de Arequipa se presentaron dos trabajos relacionados al banco de pruebas bajo normas NTP, el primero presentado por la Universidad del Centro del Perú "Laboratorio de ensayos de colectores solares térmicos y radiación solar" en donde se hace referencia a la NTP 399.400 2001 COLECTORES SOLARES: Método de ensayo para determinar la eficiencia de los colectores solares, donde mencionan el equipamiento a necesitar para determinar la eficiencia de los colectores para SCAES[1] y el otro trabajo de la Universidad Nacional de San Agustín de Arequipa [2] "Evaluación del desempeño de un banco de pruebas para sistemas de calentamiento de agua con energía solar, tipo termosifón según la norma técnica peruana NTP 399.4052007 en la ciudad de Arequipa" en donde muestran resultados realizados en un colector solar de tubos de vacío, implementaron un sistema de enfriamiento y un sistema de precalentamiento con fin de evaluar la energía acumulada por el agua.

En el XXIII SPES realizado en Huancayo se presentó el trabajo "Eficiencia de una terma solar con colector de cobre y colector CPVC para zonas altoandinas" donde se evaluó la eficiencia diaria de un SCAES para tres condiciones de radiación solar [3].

\section{METODOLOGIA}

\subsection{COLECTORES DE PLACA PLANA}

La ecuación general de eficiencia de un colector solar es [4] ecuación (1):

$$
\eta=\frac{A_{a}}{A_{g}} F_{R}(\tau \alpha)-F_{R} U_{L} \frac{\left(T_{e}-T_{a}\right)}{H}
$$

Dónde: Aa=área neta del colector solar, la parte negra, $\mathrm{m}^{2}$, Ag=área bruta del colector solar, $\mathrm{m}^{2}$, $\mathrm{F}_{\mathrm{R}}=$ factor de remoción de calor, adimensional de 0 a 1 , $(\tau a)=$ producto transmitancia absortancia, adimensional [4], $\mathrm{U}_{\mathrm{L}}=$ coeficiente de pérdidas térmicas $\mathrm{W} / \mathrm{m}^{2}-\mathrm{K}, \quad \mathrm{Te}=$ temperatura de agua al ingresar al colector, ${ }^{\circ} \mathrm{C}, \mathrm{Ta}=$ temperatura ambiente, ${ }^{\circ} \mathrm{C}, \mathrm{H}=$ radiación solar global $\mathrm{W} / \mathrm{m}^{2}$. Con la ecuación (2) se determina el producto transmitancia absortancia:

$$
(\tau \alpha)=\frac{\tau \propto}{1-(1-\alpha) \rho_{d}}
$$

Dónde: $\tau=$ transmitancia del vidrio, adimensional,

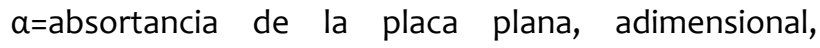
$\rho_{\mathrm{d}}=$ albedo, adimensional,

Utilizando la ecuación (3) determinamos el calor útil instantáneo [4]:

$$
Q_{u}=\dot{m} C_{p}\left(T_{s}-T_{e}\right)
$$

\subsection{CONSIDERACIONES GENERALES}

Se adiestro en el funcionamiento de los equipos cuyos resultados vamos a tomar en cuenta: los multímetros digitales, el piranometro medidor de la radiación global, cronometro digital, flujometro medidor del caudal de agua, anemómetro digital, medidor de humedad relativa.

\subsection{BANCO PARA LA EVALUACION DE EFICIENCIAS}

Para el cálculo de las eficiencias se implementó un tanque de almacenamiento de agua de 10olitros con resistencias eléctricas (4 de 1,5 KW dando un total de $6 \mathrm{KW})$. 
Se colocó un contactor para no colapsar al termostato que controlara a las resistencias, las que darán temperaturas del agua a Ta, $39^{\circ} \mathrm{C}, 59^{\circ} \mathrm{C}$ y $78^{\circ} \mathrm{C}$.

Se ubicara el piranometro (medidor de radiación solar) en el mismo plano del colector solar.

Se implementó un flujometro digital para medir el caudal de agua en GPM, un mezclador de agua caliente y fría, OFF-ON de la bomba de agua, así como válvulas. También válvulas solenoides una abierta y la otra cerrada para evaluar el flujo de masa de agua ver fig. 1.

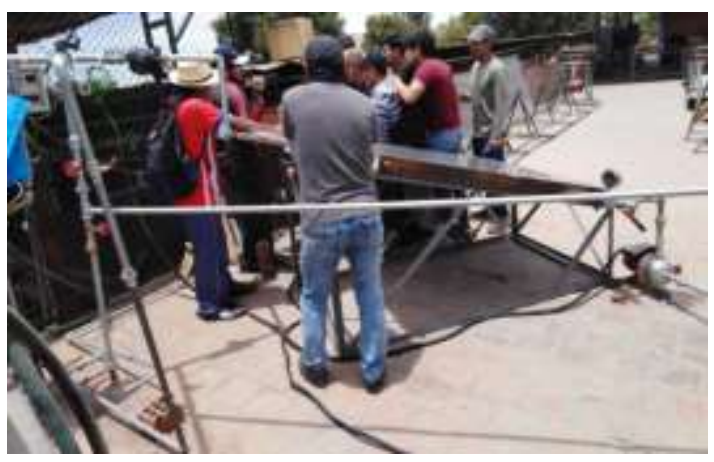

Fig. 1. Modulo estandarizado para evaluación de colectores

\subsection{EFICIENCIA}

Los datos a evaluar son: Te.

Temperatura de ingreso de agua al colector en ${ }^{\circ} \mathrm{C}$,

Temperatura de salida de agua al colector en ${ }^{\circ} \mathrm{C}$, Ts

Temperatura ambiente ${ }^{\circ} \mathrm{C}$, Ta.

Flujo de masa $\dot{m}$ de circulación por el colector de $0,02 \frac{\mathrm{Kg}}{\mathrm{s}-\mathrm{m}^{2}}$ se multiplica por el área del colector [5].

La radiación global $(\mathrm{H})$ evaluada es mayor a 790 $\mathrm{W} / \mathrm{m}^{2}[5]$.

Para cada colector se toma 8 datos antes del mediodía y 8 después del mediodía.

\section{ANALISIS DE RESULTADOS}

Calculando el calor útil instantáneo Qu y dividiendo entre la radiación incidente encontramos la eficiencia instantánea $\eta$.

Realizando las evaluaciones y graficando: $\eta$ versus (Te-Ta)/H, fig. 2.

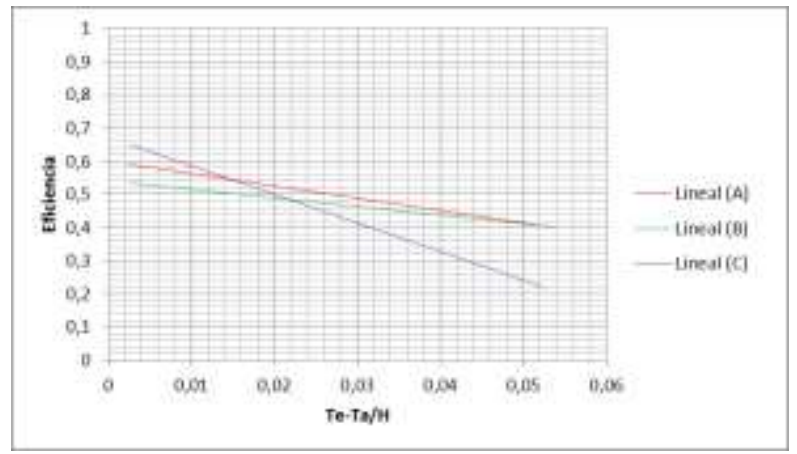

Fig. 2. Gráfica eficiencia versus (Te-Ta)/H, para los 3 diferentes colectores

DOI: https://doi.org/10.21754/tecnia.v30i1.618

\section{COLECTOR A}

\section{Eficiencia}

$\eta=0,5994-3,7047 \frac{\left(T_{e}-T_{a}\right)}{H}$

$\frac{A_{a}}{A_{g}} F R(\tau \alpha)=0,5994 \ldots \frac{1,876}{2,005} F R(\tau \alpha)=0,5994$

$F_{R}(\tau a)=0,6406$

$F_{R}\left(U_{L}\right)=3,7047 \mathrm{~W} / \mathrm{m}^{2}-\mathrm{K}$

Máxima eficiencia $\eta=59,94 \%$

Para $\eta=0 \quad \mathrm{Te}-\mathrm{Ta} / \mathrm{H}=0,162$

\section{COLECTOR B}

\section{Eficiencia}

$\eta=0,5408-2,5934 \frac{\left(T_{e}-T_{a}\right)}{H}$

$\frac{A_{a}}{A_{g}} F R(\tau \alpha)=0,5408 \ldots \frac{2,0219}{2,348} F R(\tau \alpha)=0,5408$

$$
F_{R}(\tau \alpha)=0,6280
$$

$F_{R} U_{L}=2,5934 \mathrm{~W} / \mathrm{m}^{2}-\mathrm{K}$

Máxima eficiencia $\eta=54,08 \%$

Para $\eta=0 \quad \mathrm{Te}-\mathrm{Ta} / \mathrm{H}=0,209$

\section{COLECTOR C}

\section{Eficiencia}

$\eta=0,6673-8,8343 \frac{\left(T_{e}-T_{a}\right)}{H}$

$\frac{A_{a}}{A_{g}} F R(\tau \alpha)=0,6673 \ldots \frac{2,010}{2,196} F R(\tau \alpha)=0,6673$

$F_{R}(\tau \alpha)=0,7290$

$F_{R}\left(U_{L}\right)=8,8343 \mathrm{~W} / \mathrm{m}^{2}-\mathrm{K}$

Máxima eficiencia $\eta=66,73 \%$

Para $\eta=0 \quad \mathrm{Te}-\mathrm{Ta} / \mathrm{H}=0,076$

De los 03 colectores la eficiencia máxima esta entre $54,08 \%$ a $66,73 \%$

El coeficiente global de perdidas térmicas esta entre 2,5934 a $8,8343 \mathrm{~W} / \mathrm{m}^{2}-\mathrm{K}$

$\mathrm{El}$ colector que tiene menor valor de $U_{\mathrm{L}}$ daría mayor calor útil.

\subsection{RESULTADOS}

TABLA I

Resultados de eficiencia y coeficientes globales para los 3 colectores

TECNIA Vol.30 №1 Enero-Junio 2020 


\begin{tabular}{|c|c|c|c|}
\hline COLECTOR & $\begin{array}{c}\text { П máxima } \\
\%\end{array}$ & $F_{R}(\tau \alpha)$ máximo & $\begin{array}{l}F_{R} U_{L} \text { máximo } \\
\left(W / m^{2}-K\right)\end{array}$ \\
\hline A & 59,94 & 0,6406 & 3,7047 \\
\hline B & 54,08 & 0,6280 & 2,5934 \\
\hline C & 66,73 & 0,7290 & 8,8343 \\
\hline
\end{tabular}

Los colectores a los que se hace vacío entre el vidrio y la placa absorbedora [6] y con panal de abeja transparente [7] también entre ellos, para $\eta=0$ los valores de Te-Ta/H son mayores a 0,209.

\section{CONCLUSIONES}

- De los resultados obtenidos de los colectores fabricados por tres empresas arequipeña, para colectores de $2 \mathrm{~m}^{2}$ de área absorbedora, del parque térmico de SCAES la eficiencia máxima esta entre $54,1 \%$ a $66,7 \%$, las perdidas ópticas máximas están entre $27,1 \%$ y $37,2 \%$ y el coeficiente total de transferencia de calor por factor de remoción esta entre 2,593 y $8,83\left(\mathrm{~W} / \mathrm{m}^{2}-\mathrm{K}\right)$.

- La obtención de diferentes valores de eficiencia es debido a los diferentes materiales utilizados por ejemplo vidrios, superficies selectivas y otros.

- $\quad$ El modulo estandarizado cumple con los requisitos de la norma NTP 399.4002001 al tener los instrumentos las características mínimas requeridas.

\section{RECOMENDACIONES}

- Se debería ensayar haciendo vacío entre cubierta y placa absorbedora para obtener valores más altos de eficiencia y Te-Ta/H.

- Los colectores a evaluarse deben obtener eficiencia alta y el coeficiente total de perdidas menor, ensayando vidrios de alta transmitancia y mejora en materiales aislantes.

\section{AGRADECIMIENTOS}

Este trabajo ha sido apoyado y financiado por la Universidad Nacional de San Agustín de Arequipa (UNSA), en el marco de concurso de proyectos UNSA INVESTIGA del Vicerrectorado de Investigación, proyecto con contrato $\mathrm{N}^{\circ}$ 018-2016-UNSA.

\section{REFERENCIAS}

[1] B. Camayo, C. Espinoza, W. Clemente De La Cruz, R. Massipe y M. Quispe, "Laboratorio de ensayos de colectores solares térmicos y radiación solar”, en XXII SPES Arequipa, Perú, 2015.

[2] L. Soria, A. Montoya, E. Palo y W. Cabana, "Evaluación del desempeño de un banco de pruebas para sistemas de calentamiento de agua con energía solar, tipo termosifón según la norma técnica peruana NTP 399.4052007 en la ciudad de Arequipa", en XXII SPES Arequipa, Perú, 2015.
[3] G. R. Lobato y M.A. Barrena, "Eficiencia de una terma solar con colector de cobre y colector CPVC para zonas altoandinas", en XXIII SPES Huancayo, Perú, 2016.

[4] J. A. Duffie y W. A. Beckman, Solar Engineering of Thermal Processes, USA: publicado por John Wiley \& Sons fourth ed., 2013, pp. 289-305.

[5] Indecopi, "Colectores Solares. Método de ensayo para determinar la eficiencia de los colectores solares”, NTP 399.400 2001, Perú, 2001. [6] V. Shemelin y T. Matuska, "Detailed Modeling of Flat Plate Solar Collector with Vacuum Glazing", Hindawi International Journal of Photoenergy, vol. 2017, pp. 1-9, 2017.

[7] B. P. Veinberg y V.B. Veinberg, Optics in Equipment for the Utilization of Solar Energy, Moscow, URSS: State-Publishing House of Defense Industry, 1959.

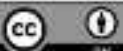

Los artículos publicados por TECNIA pueden ser compartidos a través de la licencia Creative Commons: CC BY 4.0. Permisos lejos de este alcance pueden ser consultados a través del correo revistas@uni.edu.pe 